\title{
The Effects of The COVID-19 Pandemic on Pediatric Cystic Fibrosis Patients
}

\author{
Omer Beser ${ }^{1}$, Ebru Pelin Ugur Karaboga ${ }^{1}$, EVRIM HEPKAYA ${ }^{1}$, Ayse Ayzit Kilinc ${ }^{2}$, Ahsen \\ Donmez Turkmen ${ }^{1}$, Tugce Damla Dilek ${ }^{1}$, Haluk Cokugras ${ }^{3}$, and Fügen Çullu Çokuğraş ${ }^{1}$ \\ ${ }^{1}$ Istanbul University-Cerrahpasa Cerrahpasa Faculty of Medicine \\ ${ }^{2}$ Istanbul University Cerrahpasa Faculty of Medicine \\ ${ }^{3}$ Istanbul Universitesi-Cerrahpasa
}

November 18, 2020

\begin{abstract}
Objectives: To follow-up pediatric CF patients-in terms of health status and nutritional status-via Telehealth services during the COVID-19 pandemic. Additional aims were to measure the level of anxiety in the patients and their parents, and to determine the COVID-19 transmission status in the CF patients. Materials and Methods: The CF team supported the patients via remote contact, including dedicated telephone lines. During Telehealth Services interviews, in addition to obtaining information about the patients' anthropometric measurements, health status, and CF-related complaints, the State-Trait Anxiety Inventory (STAI) was administered to the patients and the Hospital Anxiety and Depression Scale (HAD) was administered to their parents. Results: The study included 144 pediatric CF patients (74 male and 70 female). Mean age of the patients was 8.9 years. In all, $42(29.2 \%)$ of the patients were tested for COVID-19, of which 4 were positive. The mean STAI score was significantly lower in the patient group than in the control group $(\mathrm{P}<0.001)$. The mean HAD anxiety score was significantly higher in the parents of the $\mathrm{CF}$ patients, as compared to the parents of the controls $(\mathrm{P}=0.005)$. The mean HAD depression score was also statistically higher in the parents of the CF patients $(\mathrm{P}<0.001)$. Conclusion: Telehealth is an innovative method for providing healthcare services while maintaining social distancing, avoiding the risk of exposure and spread of COVID-19. Telehealth services reduce patients' and parents' anxiety, and increases their confidence in managing CF-related complications.
\end{abstract}

\section{Introduction}

The COVID-19 pandemic caused by the novel coronavirus SARS-CoV-2 has resulted in evolving public health crisis (1). Many studies have shown that the clinical manifestations of COVID-19 are primarily related to the respiratory system (2-4). Cystic fibrosis (CF) is a multi-systemic disease and the main causes of morbidity and mortality in CF patients are respiratory complications and comorbid chronic diseases (3). These problems can cause a range of psychological symptoms in pediatric CF patients and their parents (5-7). It is known that pediatric CF patients and their parents have higher depression and anxiety scores than the general population (8-10).

Viral respiratory tract infections cause more severe illness in children with $\mathrm{CF}$ than in their healthy peers. The number of $\mathrm{CF}$ patients that have been diagnosed with COVID-19 currently remains relatively low (11), making it difficult to reach conclusions about the impact of the COVID-19 infection on CF patients. In healthy individuals COVID-19 may be mild, but in individuals with significant respiratory problems the risk of more severe illness increases (11).

Respiratory infections can cause weight loss in CF patients and, therefore, tracking their nutritional status is of critical importance (12). 
The present study aimed to monitor the health status and nutritional status in pediatric CF patients during the pandemic. Additional aims were to determine the level of anxiety in the patients and their parents during the pandemic, and to determine the COVID-19 transmission status among patients.

\section{Materials and Methods}

This prospective cross-sectional study included 144 pediatric CF patients (patient group) and their parents, and 49 healthy (without any chronic and acute disease) age- and gendered-matched children (control group) and their patients. The study was performed at Istanbul University-Cerrahpaşa, Cerrahpaşa School of Medicine, Department of Pediatric Gastroenterology, Hepatology, and Nutrition, and Department of Pediatric Pulmonology, Istanbul, Turkey. Inclusion criteria included a diagnosis of CF and follow-up of [?]1 years at our clinics. Our center's CF team supported the patients via remote contact, including central email addresses and dedicated telephone lines. Regular CF appointments were conducted via Medicare Telehealth Services during the COVID-19 pandemic. The inpatient team was on site, but only saw patients face-toface when absolutely necessary. Only caveat was that it has been for MDs/dietitian/nurse only and their programmers on the list.

Approval for telework was obtained after a painstaking week of back and forth. The treatment team consisted of 2 pediatric gastroenterologists, 2 pediatric pulmonology specialists, $1 \mathrm{CF}$ dietitian, and 1 nurse that performed a mix of inpatient and outpatient work. The team was divided into 2 sub teams that alternated weekly between on-site and telework. The main responsibilities of the on-site dietitian was complete initial assessment and follow-up of patients that needed to be seen in person, including re-checking lengths/etc., formula room/emergency service discharge, daily check-ins with provider teams, and rounds [?]1 times each week with service teams The main responsibilities of the dietitian working from home were complete initial assessment and follow-up, unless the patient required being seen in person, responding to pages, notifying teams that they were teleworking, daily check-ins with provider teams, discussing pending changes to diet orders/PN with teams, contacting the on-site dietitian partner if a patient needed to be seen in person so that they could complete the assessment and provision of discharge instructions that could not be completed by a resident/RN.

As a decrease in the nutritional status in children with $\mathrm{CF}$ can be a cause of a decline in health status, the patients' parent were asked measure and their children's weight, which was then compared to the zscores last measured in the hospital. After measuring weight, height, and length of each patient, 4 z-scores were calculated using the WHO Anthro Program v.3.2.2 (January 2011) for children aged [?]5 years, as follows; height-for-age (HFA); weight-for-age (WFA); weight-for-height (WFH); body mass index-for-age (BMI). WFH in CF patients aged $<5$ years and BMI in CF patients aged $>5$ years were used to determine nutritional status.

Via the Medicare Telehealth Services the pediatric CF patients and their parents were administered a questionnaire that collected data on daily life, clinical symptoms, and medical problems between March 11 and June 01 2020. Disease flare ups, problems getting to hospital and obtaining medications, patient and family history of contact with confirmed COVID-19 cases, screen time, exercise, adherence to diet, use of vitamins, direct exposure to sunlight, and adherence to the 14 COVID-19 rules recommended by the Ministry of Health.

Medicare Health Services was used to administer the Hospital Anxiety and Depression Scale (HAD) to the parents of the patients and controls. HAD consists of 14 items, 7 of which assess anxiety and 7 of which assess depression. Cut-off values are 10 for anxiety and 7 for depression. Parents were considered positive if their score was higher than the cut-off. HAD was adapted to Turkish by Aydemir (13), who demonstrated that it is an appropriate instrument for use in Turkey. The Cronbach alpha internal consistency coefficients were 0.8525 and 0.7784 for the HAD anxiety and depression subscales, respectively. In addition, the State-Trait Anxiety Inventory (STAI) was administered to the patient and control groups to assess their mental health status. STAI was developed by Spielberger et al. (14) to measure anxiety. STAI was adapted to Turkish by Le Compte and Öner (15). The test assesses anxiety via 2 subscales: the state anxiety subscale (STAI-S) (20 
items) evaluates the current state of anxiety and the trait anxiety subscale (STAI-T) (20 items) evaluates relatively stable aspects of the tendency for anxiety, including general states of calmness, confidence, and security. The range of scores for each subscale is 20-80, with higher scores indicating greater anxiety. The cut-off point is 41 for STAI-S and 45 for STAI-T (Ercan et al. 2015). Patients were considered positive if their score was higher than the cut-off. STAI scores in the patient and control groups were compared. The Cronbach alpha internal consistency coefficients were 0.8525 and 0.7784 for STAI-S and STAI-T, respectively (13).

The correlations between the anxiety status in the CF patients and the anxiety-depression status in their parents were statistically examined. The same correlations were also analyzed in the controls and their parents. All 98 pediatric CF patients had adequate intellectual ability and education to complete STAI. The control group included 49 children because we had 49 patients aged $>8$ years. The study protocol was approved by the Istanbul University Cerrahpasa School of Medicine Ethics Committee (approval no: 29430533-604).

\section{Statistical analysis}

Data were analyzed using IBM SPSS Statistics for Windows v.21.0 (IBM Corp., Armonk, NY, USA). Associations between the study variables were measured via Pearson's chi-square test, the Mann-Whitney U test, and Student's t-test. The level of statistical significance was set at $\mathrm{P}<0.05$.

\section{Results}

\section{Descriptive findings}

The study included 144 pediatric patients (74 males and 70 female). Mean age of the patients was 8.9 years. There was a mean 4.33 individuals per patient household, with a mean age of 30.9 months. Among the patients, 15 had a decrease in appetite, of which $90 \%$ had exacerbations and $80 \%$ were hospitalized. In total, $50 \%$ of the patients (mean age: 8.81 years) had no change in appetite and (30.5\%) (mean age: 10.42 years) had an increase in appetite. There wasn't a significant difference between the age groups based on Student's t-Test.

In all, 101 of the patients did not have any CF-related symptoms, whereas 43 did, including cough $(\mathrm{n}=$ $16)$, fever $(\mathrm{n}=12)$, wheezing and shortness of breath $(\mathrm{n}=8)$, nasal obstruction $(\mathrm{n}=6)$, stomachache $(\mathrm{n}$ $=6)$, nausea $(\mathrm{n}=6)$, diarrhea $(\mathrm{n}=4)$, and constipation $(\mathrm{n}=2)$. Moreover, 22 of the patients reported via Telehealth Services that they had greasy stool. Among the patients' parents, $98.02 \%$ never considered withdrawing their children's regular medications. Among the patients, 70 came to the hospital for face to face visits, during which time their height and weight were recorded. Table 1 provides descriptive and clinical characteristics of subjects.

\section{COVID-19-positive cases}

In total, $42(29.2 \%)$ of the CF patients were tested for COVID-19, of which 4 were positive. Among those 42 patients, 28 were tested due to COVID-19 positive cases in their family, of which only 3 were positive. In all, 14 of the CF patients were tested for COVID-19 due to the presence of COVID-19 symptoms; only 1 was positive, 3 quarantined at home because they did not have any COVID-19 symptoms, and 1 was treated in hospital due to clinical symptoms of COVID-19. The characteristics of the COVID-19-positive CF patients are shown in Table 2.

\section{STAI and HAD}

STAI was administered to the $49 \mathrm{CF}$ patients aged $>8$ years. The STAI-S score in all 49 patients was above the cut-off value (normal $<45$ ). The mean STAI-S score was significantly lower in the patient group than in the control group $(\mathrm{P}<0.001)$. STAI-S scores were significantly higher in the male CF patients than in the controls and female CF patients. STAI-S scores showed that adolescent patients were more anxious than the younger CF patients and the controls. In addition, adolescents with CF had a significantly higher STAI-S score than their healthy peers and CF patients aged 8-14 years. Similarly, STAI-T scores were higher in 
the control group than in the patient group. In contrast to STAI-S findings, STAI-T scores did not differ significantly according to age in the patient group. In addition, there wasn't a significant difference in STAI-T scores between the patients and controls. STAI findings are shown in Table 3.

HAD was administered to the parents of $144 \mathrm{CF}$ patients and the parents of 140 healthy controls. The mean HAD anxiety score in the patients' parents was significantly higher than in the controls' parents.

The mean HAD anxiety score was significantly higher in the parents of the patients, as compared to the parents of the controls $(\mathrm{P}=0.005)$. The mean HAD depression score was also significantly higher in the patients' parents than in the controls' parents $(\mathrm{P}<0.001)$. Table 4 presents the HAD anxiety and depsression outcomes.

\section{Nutritional status}

The patients' BMI for age z-scores before and after the COVID-19 pandemic were compared. BMI for age and WFH z-scores in the CF patients improved significantly during the pandemic $(\mathrm{P}=0.002)$. In addition, there was significant weight gain in the patients during the pandemic $(\mathrm{P}=<0.001)$, as $74.3 \%(\mathrm{n}=107)$ of the patients gained weight. Moreover, $11.8 \%$ of the patients maintained their current weight and $13.8 \%$ lost $>3 \%$ of their weight; these patients reported having CF-related symptoms.

\section{Discussion}

The burden of COVID-19 is increasing worldwide and is thought to be greater in people with chronic diseases. On the other hand, it is predicted that COVID-19 is more aggressive in patients with CF, as it is thought that the virus affects the respiratory tract and can cause more severe disease in those with a compromised immune system. The present study aimed to monitor pediatric CF patients during the COVID-19 pandemic. The present findings show that statistically there were no problems in the supply of CF drugs or their use during the pandemic ( $\mathrm{P}=<0.001$ and $\mathrm{P}=0.001$, respectively). The present findings also show that during the pandemic there was no increase in $\mathrm{CF}$ symptom frequency or a decrease in appetite $(\mathrm{P}=0.03$ and $\mathrm{P}<0.001$, respectively). Based on these findings, we think that pediatric CF patients in Turkey are not affected by these problems during the pandemic due to well-organized protocols, such as extending the health reports of those with chronic diseases, delivering medicines to those with chronic diseases, and regular patient contact.

Studies have shown that patients with other chronic diseases are not infected to a greater degree during the COVID-19 pandemic than the general population (16). In the present study only 4 (2.7\%) of 144 pediatric CF patients were COVID-19 positive. We think that the COVID-19 positivity rate was low because as the patients had a chronic disease their parents were very careful about their isolation. Undoubtedly, we did not have enough COVID-19 positive patients to compare with those who did not; however, it was noted that among the 4 COVID-19 positive cases, 3 were infected via domestic contact and were asymptomatic. On the other hand, it was observed that the 1 symptomatic patient had both a significantly low BMI for age z-score and Pseudomonas Aeruginosacolonization.

It is clear that the COVID-19 pandemic affects individuals of all ages psychologically, which is probably related to fear and anxiety (17-19). Even though some studies have reported that patients with CF have higher levels of anxiety than healthy controls (20), CF patients are born with the disease, struggle with infections from an early age, routinely use masks, and must take medicines daily without interruption; therefore, the effects of the COVID-19 outbreak, such as wearing masks and disinfecting hands, could be expected to affect children with CF less than their healthy peers. In the present study STAI-S and STAI-T scores in the CF patient group were significantly lower than in the control group $(\mathrm{P}<0.001$ and $\mathrm{P}<0.001$, respectively), which is in accordance with earlier reports (21).

Symptoms of anxiety are more common in adolescents and adults than in the general population (22). In the present study anxiety scores were significantly higher in the 14-18-year-old CF patients. Similarly, it was reported that adolescents with non-CF bronchiectasis had higher STAI-S scores than their healthy peers (23, 24). Children with CF routinely experience exacerbation of lung symptoms, which can increase their anxiety 
level during the COVID-19 pandemic; however, high anxiety levels in healthy children may be due to the fact that they have not encountered a serious illness prior to the COVID-19 pandemic. News that Dornase alfa, which is used to treat CF, was an effective treatment for COVID-19 may have calmed children with CF. In addition, STAI-T and STAI-S scores in the present study's CF patients aged 8-14 years did not differ from those in their healthy peers, which might have been due to the fact that the patients were used to having lung problems.

In the present study HAD anxiety and HAD depression scores in the parents of the CF patients were higher than in parents of controls, as previously reported (25). As the presence of an accompanying chronic illness that primarily affects the immune and respiratory systems would likely increase the anxiety level to a greater degree in the parents of healthy children, this was an expected finding (26).

Malnutrition is an important problem in $\mathrm{CF}$ patients. It was reported that the degree of underweight in pediatric $\mathrm{CF}$ patients is an independent factor that negatively affects survival, and more recently short stature was identified as an independent risk factor for survival $(27,28)$. Among the aims of the present study was to determine the nutritional status of the CF patients via Telehealth Services and intervene as necessary. The present findings show that the CF patients improved their nutritional status during the COVID-19 pandemic $(\mathrm{P}=0.002)$. There could be 2 reasons for this. The first is that the patients attended school online due to the pandemic and spent more time at home than usual; consequently, their parents could more easily monitor their food intake. The other reason might be that the CF care team canceled face-to-face appointments to avoid unnecessary risk for the patients, but continued routine follow-up via teleconference, which reduced the patients' and parents' anxiety and increases their confidence in managing CF-related complications.

\section{Limitations}

Due to the COVID-19 pandemic, only some of the CF patients were evaluated face to face, whereas the others were monitored via Telehealth Services, which, considering the COVID-19 pandemic, was the ethical choice, as it would have been unethical to schedule unnecessary face to face evaluations due to the risk of COVID-19.

\section{References}

Guan WJ, Ni ZY, Hu Y, et al. Clinical Characteristics of Coronavirus Disease 2019 in China. N Engl J Med . 2020;382:1708-1720.

$\mathrm{Hu}$ Z, Song C, Xu C, et al. Clinical characteristics of 24 asymptomatic infections with COVID-19 screened among close contacts in Nanjing, China. Sci China Life Sci . 2020;63:706-711.

Cruz AT, Zeichner SL. COVID-19 in Children: Initial Characterization of the Pediatric Disease. Pediatrics 2020;145(6):e20200834. Accessed October 26, 2020.

Harcourt J, Tamin A, Lu X, et al. Severe Acute Respiratory Syndrome Coronavirus 2 from Patient with Coronavirus Disease, United States. Emerg Infect Dis . 2020;26:1266-1273.

O’Sullivan BP, Freedman SD. Cystic fibrosis. Lancet . 2009;373(9678):1891-1904.

Bell SC, Mall MA, Gutierrez H, et al. The future of cystic fibrosis care: a global perspective. Lancet Respir Med . 2020;8:65-124.

Pinquart M, Shen Y. Depressive symptoms in children and adolescents with chronic physical illness: an updated meta-analysis. J Pediatr Psychol . 2011;36:375-384.

Besier T, Goldbeck L. Anxiety and depression in adolescents with CF and their caregivers. J Cyst Fibros . 2011;10:435-442.

Quittner AL, Goldbeck L, Abbott J, et al. Prevalence of depression and anxiety in patients with cystic fibrosis and parent caregivers: results of The International Depression Epidemiological Study across nine 
countries. Thorax . 2014;69:1090-1097.

Sawicki GS, Rasouliyan L, McMullen AH, et al. Longitudinal assessment of health-related quality of life in an observational cohort of patients with cystic fibrosis. Pediatr Pulmonol . 2011;46:36-44.

Zachariah P, Johnson CL, Halabi KC, et al. Epidemiology, Clinical Features, and Disease Severity in Patients With Coronavirus Disease 2019 (COVID-19) in a Children's Hospital in New York City, New York. JAMA Pediatr. 2020 Jun 2020;174(10):e202430

Hauschild DB, Rosa AF, Ventura JC, et al. ASSOCIATION OF NUTRITIONAL STATUS WITH LUNG FUNCTION AND MORBIDITY IN CHILDREN AND ADOLESCENTS WITH CYSTIC FIBROSIS: A 36-MONTH COHORT STUDY. Rev Paul Pediatr . 2018;36(1):8. Published 2018 Jan 15

Aydemir, Ö., Guvenir, T., Kuey, L., et al. Validity and reliability of Turkish version of hospital anxiety and depression scale. Turk Psikiyatr. Derg. 1997;8: 280-287.

Kilic EZ, Ozguven HD, Sayil I. The psychological Effects of Parental Mental Health on Children Experiencing Disaster: The Experience of Bolu Earthquake in Turkey. FAM Process. 2003; 42:485-495.

Lecompte, A., Envanterinin, Ö.N.D.-S.K., 1975. Türkçe'ye Adaptasyon ve Standardizasyonu ile İlgili Bir Çalışma. IX. Milli Psikiyatr. ve Nörolojik Bilim. Kongresi Çalı̧̧maları 9, 457-462.

Xiao-Wei X, Xiao-Xin W, Xian-Gao J, et al. Clinical findings in a group of patients infected with the 2019 novel coronavirus (SARS-Cov-2) outside of Wuhan, China: retrospective case series. BMJ: (Online)February 2020:368.

Torales J, O'Higgins M, Castaldelli-Maia JM, Ventriglio A. The outbreak of COVID-19 coronavirus and its impact on global mental health. Int J Soc Psychiatry . 2020;66:317-320.

Brooks S, Webster R, Smith L, et al. The psychological impact of quarantine and how to reduce it: rapid review of the evidence. Lancet. 2020;395:912-920.

Havermans T, Houben J, Vermeulen F, et al. The impact of the COVID-19 pandemic on the emotional well-being and home treatment of Belgian patients with cystic fibrosis, including transplanted patients and paediatric patients. J Cyst Fibros 2020 Jul 31;S1569-1993(20)30815-8.

Havermans T, Colpaert K, Dupont LJ. Quality of life in patients with Cystic Fibrosis: association with anxiety and depression. J Cyst Fibros . 2008;7:581-584.

Pınar Senkalfa B, Sismanlar Eyuboglu T, Aslan AT, et al. Effect of the COVID-19 pandemic on anxiety among children with cystic fibrosis and their mothers. Pediatr Pulmonol . 2020;55(8):2128-2134.

Buzzi C, Tucci M, Ciprandi R, et al. The psycho-social effects of COVID-19 on Italian adolescents' attitudes and behaviors. Ital J Pediatr May 2020;46(1):69.

Yohannes AM, Willgoss TG, Fatoye FA, et al. Relationship between anxiety, depression, and quality of life in adult patients with cystic fibrosis. Respir Care 2012;57: 550-6.

Bahali K, Gedik AH, Bilgic A, et al. The relationship between psychological symptoms, lung function and quality of life in children and adolescents with non-cystic fibrosis bronchiectasis. Gen Hosp Psychiatry . 2014;36:528-532.

Cronly J, Horgan AM, Lehane E, et al. Anxiety and Depression in Parent Caregivers of Children with Cystic Fibrosis. Journal of Child and Family Studies. May 2019:28;1304-1312.

Continisio GI, Serra N, Guillari A, et al. An investigation on parenting stress of children with cystic fibrosis. Ital J Pediatr. March 2020; 46: 33.

Adde FV, Rodrigues JC, Cardoso AL. Nutritional follow-up of cystic fibrosis patients: the role of nutrition education. J Pediatr (Rio J) . 2004;80(6):475-482. 
Peterson ML, Jacobs DR Jr, Milla CE. Longitudinal changes in growth parameters are correlated with changes in pulmonary function in children with cystic fibrosis. Pediatrics . 2003;112:588-592.

\section{Hosted file}

Table 1.pdf available at https://authorea.com/users/376929/articles/493753-the-effects-ofthe-covid-19-pandemic-on-pediatric-cystic-fibrosis-patients

\section{Hosted file}

Table 2.pdf available at https://authorea.com/users/376929/articles/493753-the-effects-ofthe-covid-19-pandemic-on-pediatric-cystic-fibrosis-patients

\section{Hosted file}

Table 3.pdf available at https://authorea.com/users/376929/articles/493753-the-effects-ofthe-covid-19-pandemic-on-pediatric-cystic-fibrosis-patients

\section{Hosted file}

Table 4.pdf available at https://authorea.com/users/376929/articles/493753-the-effects-ofthe-covid-19-pandemic-on-pediatric-cystic-fibrosis-patients 